\title{
Remarks on non-BPS D-branes
}

\author{
John H Schwarz \\ California Institute of Technology, Pasadena, CA 91125, USA
}

Received 16 September 1999

\begin{abstract}
Following Sen's discovery of various stable non-BPS D-branes, K-theory has been shown to be the appropriate mathematical framework for classifying conserved D-brane charges. The classification accounts for known D-branes and predicts some new ones including a D8-brane in type I superstring theory. After briefly reviewing these developments, we discuss certain issues pertaining to the D8-brane, which is unstable.
\end{abstract}

PACS numbers: $1125,1130 \mathrm{P}$

\section{Unstable type II D-branes}

Over the past two years Ashoke Sen has pioneered the study of non-BPS D-brane systems (for reviews see $[1,2]$ ). In particular, he has focused on systems of coincident D-branes and anti-D-branes. The basic idea is that, whereas a system of coincident D-branes (or anti-Dbranes) would be a stable supersymmetric (BPS) configuration, a system with both branes and anti-branes is not. Each separately preserves half of the supersymmetries of the ambient background, but different halves are preserved in each case, so that when both are present, there is no unbroken supersymmetry. One manifestation of this fact is that the excitation spectrum of open strings connecting $\mathrm{D} p$-branes to $\overline{\mathrm{D} p}$-branes has the reversed GSO projection compared to those connecting $\mathrm{D} p$-branes to $\mathrm{D} p$-branes (or $\overline{\mathrm{D} p}$-branes to $\overline{\mathrm{D} p}$-branes). This results in tachyon fields on the worldvolume, which signals an instability. When the tachyon fields roll to a minimum-in a Higgs-like manner-this represents annihilation of branes and anti-branes.

To be specific, consider a system of $N \mathrm{D} p$-branes and $N^{\prime} \overline{\mathrm{D} p}$-branes all of which are coincident $(p+1)$-dimensional hyperplanes embedded in $\mathbb{R}^{10}$. The ground state of the $\mathrm{D} p-$ $\mathrm{D} p$ open strings gives $U(N)$ gauge fields $A$ and the $\overline{\mathrm{D} p}-\overline{\mathrm{D} p}$ open strings give $U\left(N^{\prime}\right)$ gauge fields $A^{\prime}$. The $\mathrm{D} p-\overline{\mathrm{D} p}$ open strings, on the other hand, give a bifundamental $\left(N, N^{\prime}\right)$ tachyon $T$. These fields can be written together as a 'superconnection'

$$
\mathcal{A}=\left(\begin{array}{cc}
A & T \\
\bar{T} & A^{\prime}
\end{array}\right) .
$$

One issue is whether or not the branes and anti-branes can completely annihilate. The criterion, basically, is whether the total D-brane charge (which is conserved) is zero or not. Cancelling the $\mathrm{D} p$-brane charge requires $N=N^{\prime}$, of course, but that is not the whole story. It is also necessary that the gauge bundles $E$ and $E^{\prime}$ (associated with branes and anti-branes) should be topologically equivalent, $E \sim E^{\prime}$. Otherwise, there is some lower-dimension D-brane charge, and such a D-brane would survive. To illustrate this, consider the case of one D2-brane and 
one $\overline{\mathrm{D} 2}$-brane, which are wrapped on a $T^{2}$, and coincident in the other dimensions. The Wess-Zumino term of the D2-brane worldvolume action contains

$$
\int\left(C \mathrm{e}^{F}\right)_{3}=\int_{R \times T^{2}}\left(C_{3}+C_{1} \wedge F\right),
$$

where the $C$ 's are $R R$ potentials. From this formula we see that the magnetic flux $\int_{T^{2}} F$ is a source of $C_{1}$, which means that it carries D0-brane charge. Thus, for example, if the D2brane has flux giving one unit of D0-brane charge and the $\overline{\mathrm{D} 2}$-brane has no such flux, then the annihilation leaves a D0-brane

$$
\mathrm{D} 2+\overline{\mathrm{D} 2} \rightarrow \mathrm{D} 0 .
$$

The worldvolume theory that describes a coincident $\mathrm{D} p+\overline{\mathrm{D} p}$ system can be formulated in terms of the gauge fields and tachyons, where one imagines that all other modes have been integrated out. It is hard to make this explicit in a controlled manner, since the tachyon mass is generally of string scale. Thus the discussion that follows is necessarily somewhat qualitative and heuristic. It does have the advantage of being very physical and intuitive, however. Analyses with better mathematical control lead to the same conclusions. One approach is to use conformal field theory methods, as described by Sen. Another approach is to use boundary-state techniques, as described by Gaberdiel. In any case, working with gauge fields and tachyons, the worldvolume theory has a tachyon potential $V(T)$, which must be invariant under the $U(N) \times U\left(N^{\prime}\right)$ gauge symmetry. Moreover, when $N=N^{\prime}$, Sen argues that it should have minima that correspond to pure vacuum. The locus of minima, all of which are gauge equivalent, is given by $T=T_{0} \mathcal{U}$, where $T_{0}$ is a fixed positive real number and $\mathcal{U}$ is an arbitrary constant element of $U(N)$. At the minimum, the tachyon condensation energy should exactly cancel the energy of the D-branes

$$
V\left(T_{0} \mathcal{U}\right)+2 N T_{\mathrm{D} p}=0 .
$$

Here, $T_{\mathrm{D} p}$ is the tension of a single $\mathrm{D} p$-brane. Thus when $E \sim E^{\prime}$ and $T=T_{0} \mathcal{U}$, the $\mathrm{D} p+\overline{\mathrm{D} p}$ system is equivalent to pure vacuum. What happens to the $U(N)$ gauge groups is not completely understood.

Let us now take $N=N^{\prime}=1$ and consider a kink configuration of the tachyon field $T$. $T$ is complex, so let us consider $\operatorname{Im} T=0$ and $\operatorname{Re} T=T_{0} \tanh (x / a)$, where $x$ is one of the Cartesian coordinates on the branes. This describes a solitonic $\mathrm{D}(p-1)$-brane of thickness $a$ concentrated in the vicinity of $x=0$. (The precise functional form is not important.) Since the vacuum manifold $|T|=T_{0}$ is a circle, and $\pi_{0}\left(S^{1}\right)$ is trivial, this D-brane has a real tachyon in its worldvolume and is unstable. This is just as well, since the stable D-branes of type II theories are believed to be known, and this one is not on the list. In fact, such unstable D-branes can be constructed for all 'wrong' values of $p$ in type II theories. Stable D-branes exist for $p=$ even in the IIA theory and $p=$ odd in the IIB theory. The unstable ones occur for the other values of $p$. Sen has demonstrated that these unstable D-branes are useful for analysing certain issues. My purpose in describing them here is to set the stage for an analogous construction, which will appear later.

\section{Non-BPS type I D0-branes}

Let me now review one of Sen's constructions of a non-BPS stable D0-brane in type I superstring theory. The construction we will consider is in terms of a tachyon kink in a Dstring-anti-D-string configuration. Recall that the type I D-string is actually the $\operatorname{Spin}(32) / \mathbb{Z}_{2}$ heterotic string continued to strong coupling. The continuation is reliable because the string is BPS. A system of $N$ coincident $D$ strings has worldvolume gauge group $O(N)$. This can 
be understood as the subgroup of $U(N)$ on a set of type IIB D-strings that survives orientifold projection. In particular, for a single D-string the group is $O(1)=\mathbb{Z}_{2}$. Even though there are no gauge fields in this case, the group matters. In particular, a D-string wrapped on a circular spatial dimension has possible Wilson lines $W= \pm 1$.

The 32 left-moving fermion fields $\lambda^{A}$ on the D-string worldsheet arise as zero modes of D1-D9 open strings. When wrapped on a circular dimension, the Wilson line encodes their periodicity

$$
\lambda^{A}(x+2 \pi R)=W \lambda^{A}(x) .
$$

Thus, for $W=1, \lambda^{A}$ has zero modes, which satisfy a Clifford algebra, and D-string quantum states are gauge group spinors (with $2^{15}$ components).

Now consider a D1 $+\overline{\mathrm{D} 1}$ pair wrapped on a circle. If one string has $W=1$ and the other one has $W=-1$, then the overall two-particle state is a gauge group spinor. Since the gauge group is not broken, this implies that complete annihilation is not possible. The tachyonic ground state of the open string connecting the D-string and the anti-D-string is real in this case. For the case of opposite Wilson lines that we are considering, the tachyon field is antiperiodic. Thus it has the Fourier series expansion

$$
T=\sum_{n} T_{n+1 / 2}(t) \exp \left[\mathrm{i}\left(\frac{n+\frac{1}{2}}{R}\right) x\right] .
$$

The mass of $T_{n+1 / 2}$, considered as a particle in nine dimensions, is

$$
M_{n+1 / 2}^{2}=\left(n+\frac{1}{2}\right)^{2} / R^{2}-\frac{1}{2} .
$$

The $-\frac{1}{2}$ term is the tachyonic mass-squared value (in string units) in ten dimensions, as usual for an RNS string. From this formula we see that for $R<1 / \sqrt{2}$, there is no tachyonic instability and the wrapped $\mathrm{D} 1+\overline{\mathrm{D} 1}$ pair does not annihilate. For $R>1 / \sqrt{2}$, on the other hand, $T_{ \pm 1 / 2}$ (and possibly other modes) are tachyonic. This means that the strings can annihilate. What results is a stable non-BPS D0-brane, which is a gauge group spinor. It carries a conserved $\mathbb{Z}_{2}$ charge. In this case, the $\mathbb{Z}_{2}$ corresponds to the two conjugacy classes of $\operatorname{Spin}(32) / \mathbb{Z}_{2}$.

At $R=R_{c}=1 / \sqrt{2}$ and small string coupling constant $g$

$$
M_{\mathrm{D} 0} \sim 2 \times 2 \pi R_{c} \times T_{\mathrm{D} 1}=\sqrt{2} / g .
$$

Sen has argued that this is the leading small $g$ value of the type 1 D0-brane mass for all $R$, though there are higher-order corrections. It has the usual $1 / g$ factor that is characteristic of D-branes. Curiously, its mass differs from that of the type IIA D0-brane by a factor of $\sqrt{2}$ (in leading order). In the S-dual heterotic theory the lightest gauge group spinor occurs at the first excited level in the perturbative spectrum. Presumably, the non-BPS D0-brane of type I is this state continued to strong coupling.

\section{K-theory classification of D-branes}

Recall that a $\mathrm{D} p+\overline{\mathrm{D} p}$ system is characterized by a pair of vector bundles $\left(E, E^{\prime}\right)$ and a tachyon $T$, which is a section of $E^{\star} \otimes E^{\prime}$. Complete annihilation should be possible if and only if $E \sim E^{\prime}$. This requires $N=N^{\prime}$, in particular. Following an earlier suggestion by Moore and Minasian [3], Witten has argued that equivalence classes of pairs $\left(E, E^{\prime}\right)$ that can be related by brane annihilation and creation correspond to K-theory classes [4]. So these are the mathematical objects that correspond to conserved D-brane charges. 
For example, D-brane charges of the type IIB theory on $\mathbb{R}^{10}$ are given by

$$
\tilde{K}\left(S^{9-p}\right)= \begin{cases}\mathbb{Z} & p=\text { odd } \\ 0 & p=\text { even. }\end{cases}
$$

This accounts for the RR charges of all stable type IIB D-branes. Note that the unstable D-branes (for $p=$ even) carry no conserved charges and do not show up in this classification.

In the case of type I theory, $E$ is an $O(N+32)$ bundle and $E^{\prime}$ is an $O(N)$ bundle, so that the total $\mathrm{RR}$ 9-brane charge is 32 . The relevant K-theory groups for $\mathbb{R}^{10}$ in this case are denoted by $\widetilde{K O}\left(S^{9-p}\right)$, as explained by Witten.

The results are as follows:

- $\widetilde{K O}\left(S^{9-p}\right)=\mathbb{Z}$ for $p=1,5,9$

these classify the charges for the three kinds of BPS Dp-branes of type I.

- $\widetilde{K O}\left(S^{9-p}\right)=\mathbb{Z}_{2}$ for $p=-1,0,7,8$

$p=-1$ corresponds to the type I D-instanton, and $p=0$ corresponds to the non-BPS D0-brane, which we have discussed. The cases $p=7,8$ are additional non-BPS D-branes proposed by Witten.

- $\widetilde{K O}\left(S^{9-p}\right)=0$ for $p=2,3,4,6$

there are no conserved D-brane charges in these cases.

\section{Issues concerning the type I D8-brane}

The K theory classification of type I D-branes, which we have just summarized, suggests two new D-branes not discussed previously: D7 and D8, each of which is supposed to carry a conserved $\mathbb{Z}_{2}$ charge.

As noted in the final paragraph of [5], there is a tachyon in the spectrum of D7-D9 and D8-D9 open strings. This means that the worldvolume of a D7- or D8-brane contains 32 tachyon fields. Therefore, neither of these D-branes is stable. This raises the question of what happens to the conserved $\mathbb{Z}_{2}$ charge when they dissolve into the background D9-branes. The comments that follow arose out of discussions with Oren Bergman and Ashoke Sen, as well as correspondence with Edward Witten. I will only discuss the D8-brane, though the D7-brane story is likely to be similar.

Witten has argued in support of the D8-brane as follows. The type I D-instanton implies that there are two different 'vacua', distinguished by the sign of the instanton amplitudes. This is a $\mathbb{Z}_{2}$ analogue of the $\theta$ angle in QCD. One should expect that there is a domain wall separating the two vacua and this should be the D8-brane. The sign change of instanton amplitudes would mean that the D-instanton is the electromagnetic dual of the D8-brane. Investigations that support this picture were carried out by Gukov [6].

The K-theory analysis incorporates Bott periodicity. This suggests that the type ID8-brane should have features in common with the type I D0-brane, discussed in section 2. Of course, Bott periodicity should be taken cum grano salis, since the total spacetime dimension is ten. The construction of the type I D0-brane that we described involved wrapping D-strings on a circle, which was a convenient regulator. However, one might argue that a localized D8-brane should not exist on a circle (in the direction normal to the brane), since this would require identifying the two distinct vacua. Therefore, we will analyse the situation in uncompactified $\mathbb{R}^{10}$.

The D0-brane could have been presented without involving compactification. In any case, by considering the $R \rightarrow \infty$ limit of the construction in section 2, we see that the D0-brane can be described as a tachyonic kink in a system consisting of an infinite straight D-string and 
a coincident anti-D-string. The kink configuration would be exactly the same as we described for type II theories in section 1. However, unlike the type II examples, the tachyon field is real in this case, and the potential $V(T)$ is an even function because of the $\mathbb{Z}_{2}$ gauge symmetries. The kink configuration describing the D0-brane is topologically stable in this case because the vacuum manifold is $S^{0}\left(T= \pm T_{0}\right)$ and $\pi_{0}\left(S^{0}\right)=\mathbb{Z}_{2}$.

Let us now try to construct the D8-brane out of D9-branes in an analogous manner. One essential difference is that the total D9-brane charge must be 32. Therefore, the simplest analogue to consider is $33 \mathrm{D} 9$-branes and one $\overline{\mathrm{D} 9}$-brane filling the entire $\mathbb{R}^{10}$ spacetime. In this case the open strings connecting the $\overline{\mathrm{D} 9}$-brane to the $\mathrm{D} 9$-branes give 33 real tachyon fields $\vec{T}$ in the fundamental representation of $S O(33)$. (It does not matter whether one uses $O(N)$ or $S O(N)$ in the present setting.) The potential $V(\vec{T})$ must have $S O(33)$ symmetry and therefore the vacuum manifold should be given by $|\vec{T}|=T_{0}$, which describes an $S^{32}$. This manifold is connected, so there is no topologically stable kink. This is the same situation we encountered for the unstable type II D-branes in section 1. In this case there are 32 directions of instability, so one expects to find 32 tachyon fields in the D8-brane worldvolume. This agrees with the conclusion of [5], which identified them with modes of D8-D9 open strings.

So what are we to make of all of this? I think it is clear that the D8-brane is unstable, at least unless something further is done to stabilize it. Still, it may be interesting to consider setting up a D8-brane configuration and exploring what that implies. I will not present the details of the reasoning here, but it appears that the vacua on the two sides of the D8-brane are distinguished by the chirality of gauge group spinors. Of course, once the D8-brane decays, eventually leaving a uniform type I vacuum, only one chirality will remain. This may sound paradoxical, but it is possible because the gauge group is broken inside the D8-brane.

In conclusion, K-theory classifies D-brane charges. However, high-dimensional non-BPS D-branes are sometimes destabilized by tachyonic modes of open strings connecting them to background spacetime filling D-branes.

\section{Acknowledgments}

I am grateful to Oren Bergman, Ashoke Sen and Edward Witten for helpful discussions and suggestions. This work was supported in part by the US Department of Energy under grant no DE-FG03-92-ER40701.

\section{References}

[1] Sen A 1999 Preprint hep-th/9904207

[2] Lerda A and Russo R 1999 Preprint hep-th/9905006

[3] Moore G and Minasian R 1997 J. High Energy Phys. JHEP11(1997)002 (Moore G and Minasian R 1997 Preprint hep-th/9710230)

[4] Witten E 1998 J. High Energy Phys. JHEP12(1998)019 (Witten E 1998 Preprint hep-th/9810188)

[5] Frau M, Gallot L, Lerda A and Strigazzi P 1999 Preprint hep-th/9903123

[6] Gukov S 1999 Preprint hep-th/9901042 